\title{
Effect of grain boundary characteristics on hot tearing in directional solidification of superalloys
}

\author{
Yizhou Zhou, Andreas Volek, and Robert F. Singer ${ }^{\text {a) }}$ \\ WTM Institute, Department of Materials Science, University of Erlangen-Nürnberg, \\ 91058 Erlangen, Germany
}

(Received 27 February 2006; accepted 19 May 2006)

The effect of grain boundary (GB) misorientation on hot tearing susceptibility of directionally solidified (DS) nickel-based superalloys was explored. We found that the castability of second generation nickel-based superalloy CMSX-4 is inferior to DS superalloy IN792, an alloy well known for bad castability. The castability of CMSX-4 is somewhat improved at a higher solidification rate. The hot tearing tendency increases with increasing GB misorientation angle. As feeding tendency becomes greater with increasing misorientation, this points to the importance of GB cohesion for solidification cracking in the alloy. Microstructure investigation reveals that hot tearing is associated with formation of continuous gamma and gamma prime eutectic films at the GB in CMSX-4. We assume that the gamma and gamma prime eutectic, which reflects the remaining liquid at the end of solidification, prevents the impinging dendrite arms from touching and in this way decreases cohesion.

\section{INTRODUCTION}

Single crystal (SC) turbine blades produced by directional solidification (DS) have led to dramatic improvements in gas turbine performance. ${ }^{1}$ Recent developments in SC superalloy compositions have aimed at the further increase of temperature capability. Progress was made by increasing refractory element additions such as tantalum $(\mathrm{Ta})$, rhenium $(\mathrm{Re})$, and tungsten $(\mathrm{W})$ because of their important solid solution strengthening effect. However, the casting characteristics of these newer high-refractory alloys are significantly worse than those of the earlier alloys. Pollock et al. ${ }^{2-4}$ reported refractory alloying additions to promote the formation of freckle-type defects. McLean et al. ${ }^{5-7}$ found that the high-refractory superalloys yield a wider range of crystal orientations. The present article studies hot tearing susceptibility of these alloys.

Crack formation along longitudinal grain boundaries (GBs) during the last stages of solidification is a wellknown problem in a DS nickel-based superalloy. The phenomenon is known as hot tearing, hot cracking, or solidification cracking in contrast to cold cracking or stress relief cracking, which occurs below the solidus temperature during further cooling. Hot tearing phenomena as a source for bad castability has been studied

\footnotetext{
${ }^{a}$ Address all correspondence to this author. e-mail: robert.singer@ww.uni-erlangen.de DOI: 10.1557/JMR.2006.0284
}

extensively over the past few decades. ${ }^{8} 9$ It is generally believed that hot tearing occurs in a so-called "film stage" at the last stages of solidification where the residual liquid is still more or less continuous, and that hot tearing is a GB phenomena: the initiation or propagation of hot cracks is usually intergranular. ${ }^{10,11}$ In the case of a DS process where hot tears develop along the broad faces of columnar grains, this means that only small GB cohesion forces are developed, essentially by the bridging of secondary dendrite arms. If this small amount of resistance is overcome by tensile forces because the volume contraction during solidification and feeding is impeded, hot tearing will occur.

In our previous work, ${ }^{12}$ the effects of GB misorientation on castability were examined by casting $\mathrm{SC}$ and bi-crystal (BC) samples. It was found that the hot tearing susceptibility of DS superalloy IN792 is a function of the GB misorientation, i.e., the hot tearing tendency of the alloy increases with increasing GB misorientation. As feeding can only be improved with increasing GB misorientation, the increasing susceptibility to hot tearing must be a consequence of the reduced cohesion strength that is probably caused by reduced secondary arm bridging. Reduced bridging occurs as there are geometrical constraints at the GB or as more prominent liquid films are formed at the GB. These results are well in accordance with the general view of hot tearing and they show the nature of the residual liquid at the GB to play an important role during the formation of hot cracks.

In another investigation, ${ }^{13}$ we found that solidification 
rate is able to affect the geometry and distribution of remaining liquid, although the volume fraction of the remaining liquid is independent of the solidification rate. The remaining liquid becomes more finely dispersed and increasingly discontinuous at a higher solidification rate because of the refinement of dendrites and columnar grains. Because there are larger bridging areas as the continuous liquid film is avoided, the strength of the solidifying skeleton is increased and the hot tearing tendency is reduced.

The aim of the present work was to explore the hot tearing susceptibility of high-refractory superalloy CMSX-4 as a function of the GB misorientation in a similar vein as in Ref. 12. The comparison of CMSX-4 and IN792 is helpful in understanding the hot tearing performances in both alloys. Furthermore, different solidification rates are used to change dendrite arm spacing of CMSX-4 SC and BC castings in the present work so that the effect of microstructure parameters on hot tearing susceptibility can be studied.

In our previous work, ${ }^{13}$ the nucleation at the start of DS process was controlled by the casting parameters (i.e., the temperature of melt and chill plate), not by a BC seeding procedure, as in the present article. Therefore, dendrite arm spacing and columnar grain size were reduced simultaneously in the previous work ${ }^{13}$ when the solidification rate was increased. As such, the effects of the dendrite arm spacing and columnar grain size on castability could not be studied separately. In the present work, because the columnar grain number is constant (SC or BC), the effect of dendrite arm spacing on castability can be studied alone as the solidification rate is varied.

Superalloy CM247 was chosen as a reference material in our previous works ${ }^{12,14,15}$ because of its well-known good castability. The microstructural characteristics of this alloy are recalled in the present work to obtain better insight into the relationship between the microstructural parameters and castability.

\section{EXPERIMENTS}

\section{A. Castability tests}

The material used in the present work was supplied by Doncasters Precision Castings (Bochum, Germany). Table I lists the composition in wt\%.
To test castability or hot tearing susceptibility, tubelike castings were produced and visually inspected for crack formation after solidification. SC seeds from superalloy CMSX-4 and PWA1483 were used to initiate the development of CMSX-4 and IN792 SC or BC tube castings, respectively. In our previous work, ${ }^{12}$ it was reported that PWA1483 seeds do not influence the nominal composition of IN792 after casting because PWA1483 is the SC equivalent of IN792 having almost the same composition except the minor elements such as $\mathrm{B}$ and $\mathrm{Zr}$.

Machined SC seeds were placed at the bottom of the mold as shown in Fig. 1. The [001] direction of each SC seed is well parallel to the ideal [001] DS direction (the [001] deviation angle of each SC seed from the ideal [001] direction is less than $2^{\circ}$ ). For simplification, therefore, we assume that all columnar grains in the samples produced by such a seed technique have an exact [001] orientation parallel to the mold axis. The misorientation between two grains can then be described by the angle of rotation around the [001] axis. The [100] or [010] orientations of SC seeds were prearranged in such a way that GB misorientation of the final castings could be controlled. The same techniques were used to produce SC and BC samples in our previous work. ${ }^{12}$ It should be emphasized that the material and geometry of the alumina tubes used as molds are exactly the same in the present work as in our earlier work, ${ }^{12}$ i.e., the tube-like castings have exactly the same inner and outer diameters as well as wall thickness in both works. The only difference is that the inner alumina is placed at a higher position in the present work, and this change in the test set-up does not influence the level of hot tearing, as will be seen later.

Castability tests were performed by a standard withdrawal method. The alloy was heated to $1550{ }^{\circ} \mathrm{C}$ and was kept for $5 \mathrm{~min}$ before it was poured into the preheated mold (mold temperature, $1500{ }^{\circ} \mathrm{C}$ ). Two different speeds of 1 and $6 \mathrm{~mm} / \mathrm{min}$ were chosen for withdrawal of the CMSX-4 castings from the furnace. The withdrawal speed for the castings of IN792 was $1 \mathrm{~mm} / \mathrm{min}$ only. The same mold temperature $\left(1500{ }^{\circ} \mathrm{C}\right)$ and withdrawal speed $(1 \mathrm{~mm} / \mathrm{min})$ were used to cast IN792 in the previous work. $^{12}$

Stresses arise during solidification and further cooling because of different thermal contractions of the alloy and

TABLE I. Nominal compositions of alloys as used in the experiments (in weight percent).

\begin{tabular}{|c|c|c|c|c|c|c|c|c|c|c|c|c|c|}
\hline Alloy & $\mathrm{Cr}$ & $\mathrm{Co}$ & Mo & $\mathrm{W}$ & $\mathrm{Al}$ & $\mathrm{Ti}$ & $\mathrm{Ta}$ & $\mathrm{Re}$ & $\mathrm{Hf}$ & B & $\mathrm{Zr}$ & $\mathrm{C}$ & $\mathrm{Ni}$ \\
\hline CMSX-4 & 6.5 & 9.0 & 0.6 & 6.0 & 5.6 & 1.0 & 6.5 & 3.0 & 0.1 & $\ldots$ & $\ldots$ & $\ldots$ & Balance \\
\hline IN792 & 12.5 & 8.8 & 1.8 & 4.0 & 3.4 & 3.9 & 3.9 & $\ldots$ & $\ldots$ & 0.014 & 0.017 & 0.08 & Balance \\
\hline PWA1483 & 12.2 & 9.0 & 1.9 & 3.8 & 3.6 & 4.2 & 5.0 & $\cdots$ & $\cdots$ & $\cdots$ & $\cdots$ & 0.07 & Balance \\
\hline CM247 & 8.0 & 9.4 & 0.5 & 9.5 & 5.7 & 0.7 & 3.2 & $\ldots$ & 1.5 & 0.017 & 0.018 & 0.07 & Balance \\
\hline
\end{tabular}




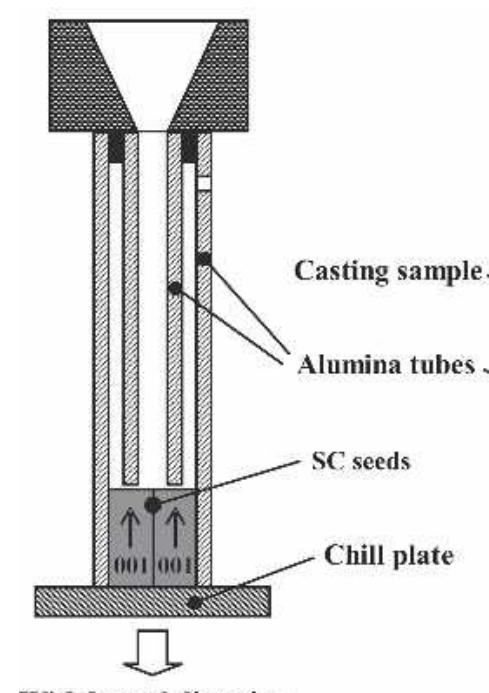

Withdrawal direction

(a)

FIG. 1. Schematic illustration of the castability test used in the present work. The melt is poured into the tube-shaped mold and subsequently solidified by withdrawal of the mold from the furnace. (a) Alumina mold with SC seeds at the bottom, (b) top view of seeds to grow BC samples, and (c) dimensions of castings. The [001] direction of each SC seed is exactly parallel to the [001] DS direction. White crosses in (b) are meant to symbolize dendrite cores or [100] and [010] directions, respectively.

the alumina mold. The stresses occurring in this specific castability test were severe compared with those of a casting using standard mold material because the alumina core tube does not yield at all. (It contains a much lower silica content and lower porosity than the ceramic used for molds in commercial DS.)

The castability of castings was evaluated by measuring a "crack ratio" obtained from evaluation of the length and width of the cracks. The crack ratio $r$ is defined as

$$
r=\frac{\sum_{i=1}^{n} l_{i} w_{i}}{L C}
$$

where $n$ is the number of cracks, $l_{i}$ and $w_{i}$ are the length and the maximum width of a crack, respectively, $L$ is the length of the casting tube (the length of the seeds is not included in $L$ ), and $C$ is the outer circumference of the tube.

\section{B. Sample examination}

GB misorientation angle $(\alpha)$, primary dendrite arm spacing $\left(\lambda_{1}\right)$, secondary dendrite arm spacing $\left(\lambda_{2}\right)$, and volume fraction of $\gamma / \gamma^{\prime}$ eutectic were determined by optical metallography on etched samples cut perpendicular to the solidification direction using an image analysis system with OPTIMAS 6.2 software (Optimas Corporation, Bothell, WA). The GB misorientation $\alpha$ was measured from secondary dendrite arm orientations. It was reported in our previous work ${ }^{12}$ that the measurement for $\alpha$ from secondary dendrite arm orientations by optical microscopy is practical and that the accuracy of this form of measurement is high enough for the present work. The primary dendrite arm spacing $\lambda_{1}$ was determined by counting the number $\left(n_{1}\right)$ of dendrites within a known area (A) and using the equation: $\lambda_{1}=\left(\mathrm{A} / \mathrm{n}_{1}\right)^{1 / 2}$, i.e., dendrites are assumed to be square shaped. The secondary dendrite arm spacing $\lambda_{2}$ was determined by counting the number $\left(\mathrm{n}_{2}\right)$ of secondary dendrite arms along a straight line with a known length (1) and using the equation: $\lambda_{2}=1 / n_{2}$. The straight line is parallel to the DS direction and 1 is as long as possible to reduce the experimental error. The fraction of $\gamma / \gamma^{\prime}$ eutectic was always measured within the same frame area, irrespective of whether it did or did not contain a GB. It is generally accepted that the "area fraction" of a phase is equal to its "volume fraction."16,17 A scanning electron microscope (SEM; XL30; Philips, Eindhoven, The Netherlands) was used for detailed microstructure observation.

During the sample examination, a number of IN792 and CM247 samples from our previous work ${ }^{12}$ were reexamined.

\section{RESULTS}

\section{A. Castability tests}

The castability of CMSX-4 was found to be rather bad and even the SC castings displayed hot tearing (Fig. 2). The dendrites in the metallographic sample cut perpendicular to the solidification direction appear as bright crosses in Fig. 2(a). The [100] or [010] direction can be determined from the orientation of secondary dendrite arms. It is apparent that the crack in the figure is an interdendritic crack and the secondary dendrite arms on both sides of the crack are identical in orientation. The typical fracture surface from the crack is shown in Fig. 2(b). No cleavage planes that are typical for crack formation in the solid state are evident. Therefore, we conclude that the cracks in CMSX-4 SC castings are hot cracks.

The castability of CMSX-4 SC and BC castings solidified at $1 \mathrm{~mm} / \mathrm{min}$ is summarized in Fig. 3(a). The data of IN792 castability tests from the previous work ${ }^{12}$ and the present work are also included in Fig. 3(a). The two data points for crack ratios in IN792 from the present work show good agreement with previous data; ${ }^{12}$ this demonstrates that the present experimental set-up and method of data analysis is well in accordance with the previous work. IN792 shows hot cracking at high GB misorientations, whereas it is free of hot cracking when the GB misorientation is less than $12^{\circ}$. Because CMSX-4 


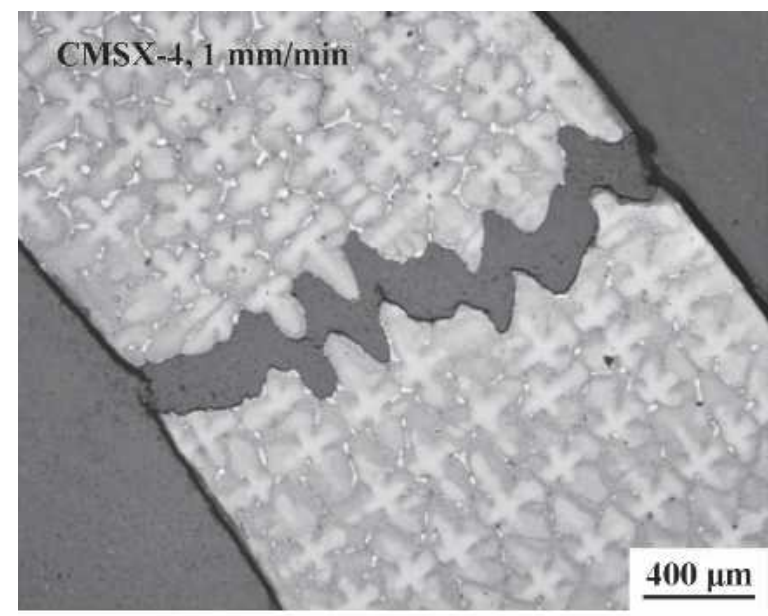

(a)

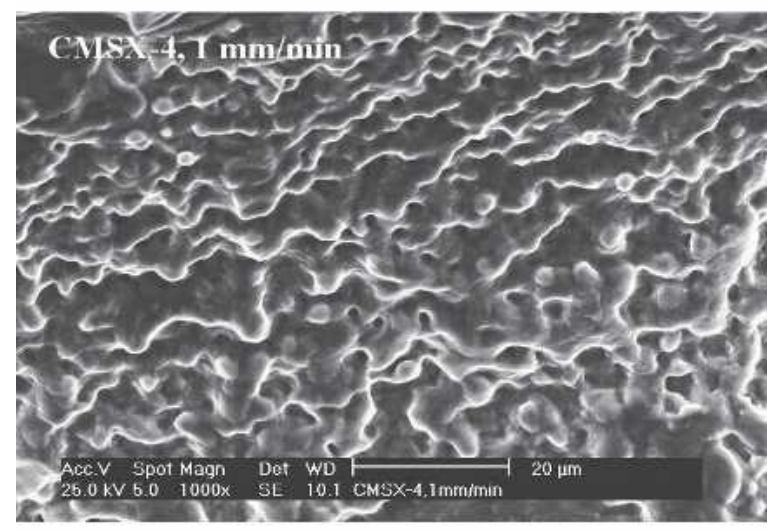

(b)

FIG. 2. Hot cracking in a CMSX-4 SC casting solidified at $1 \mathrm{~mm} / \mathrm{min}$. (a) Optical micrograph showing the propagation route of the crack and (b) SEM image showing the fracture surface of the crack. The dendrites in the metallographic sample cut perpendicular to the solidification direction appear as bright crosses. The crack is an interdendritic crack and the orientations of secondary dendrite arms on both sides of the crack are identical, as evidenced by the measurement for secondary dendrite arm orientations under optical microscopy. On the fracture surface, no cleavage planes that are typical for crack formation in the solid state are visible.

is not free of hot cracking at low levels of GB misorientation, it can be concluded that the castability of CMSX-4 is inferior to IN792.

The hot tearing susceptibility of CMSX-4 is reduced at higher solidification rate. Figure 3(b) allows a comparison of the castability of CMSX-4 solidified at 1 and $6 \mathrm{~mm} / \mathrm{min}$. CMSX-4 castings solidified at $6 \mathrm{~mm} / \mathrm{min}$ are free of hot cracking when the GB misorientation is less than $11^{\circ}$. Small cracks are observed when the GB misorientation increases to $14^{\circ}$. Large GB misorientation (over $20^{\circ}$ ) leads to the formation of large cracks, and the crack ratios at great GB misorientation are more or less the same at both solidification rates.

The castability of CM247 is very good, and the alloy is always free of hot cracking under conditions where IN792 is not. $^{12,14,15}$
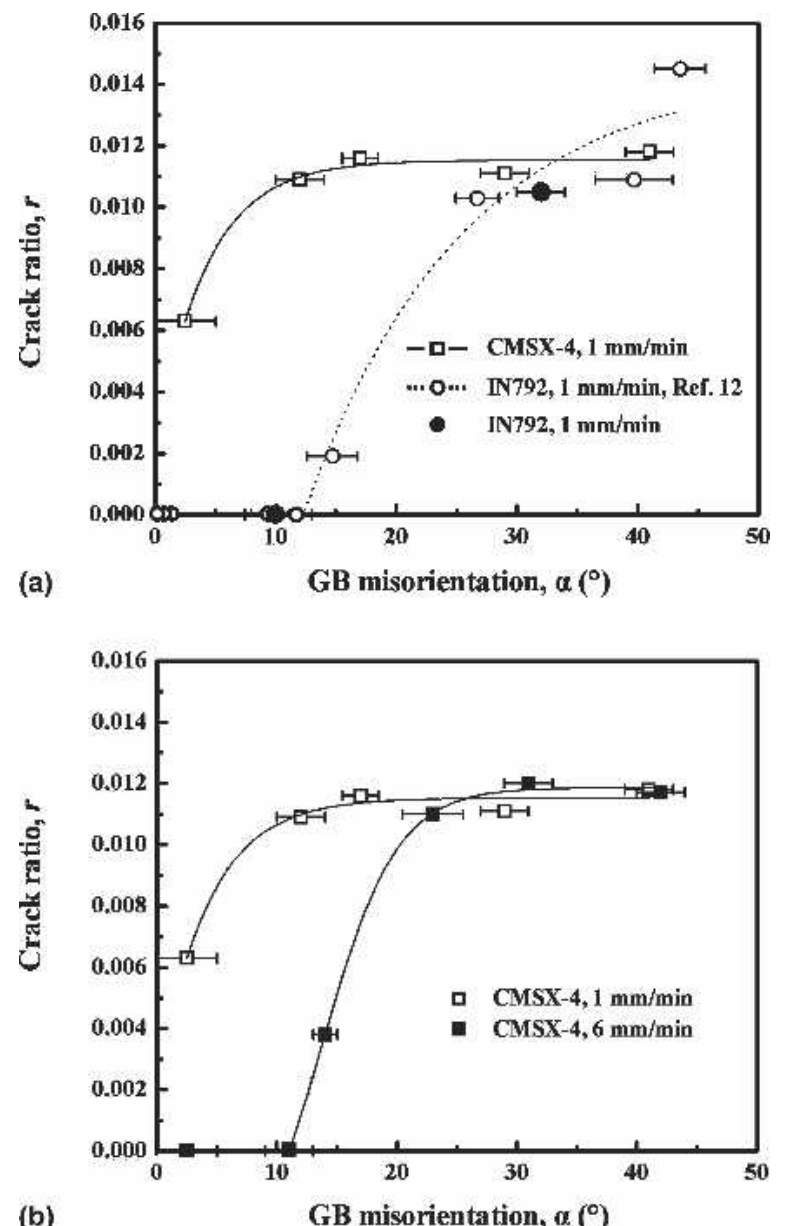

FIG. 3. Crack ratio $r$ as a function of GB misorientation. (a) Comparison of CMSX-4 and IN792 at the same solidification conditions and (b) comparison of CMSX-4 at different solidification rates.

\section{B. Microstructure characterization}

Data for the primary dendrite arm spacing $\lambda_{1}$ of CMSX-4, IN792, and CM247 is presented in Fig. 4. It is clear from the figure that $\lambda_{1}$ of CMSX-4 is smaller at higher solidification rate. The result is well in accordance with the literature (see Ref. 18). Because $\lambda_{1}$ increases with increasing distance from the chill end-caused by a change in the thermal gradient during solidification in the set up used-the following microstructure characterization refers always to a cut of the sample at a fixed distance of $60 \mathrm{~mm}$ from the chill end.

Microstructure characterization indicates that there is a relation between castability and $\gamma / \gamma^{\prime}$ eutectic formation in CMSX-4 and IN792. In all castings of both alloys without hot cracking, the $\gamma / \gamma^{\prime}$ eutectic is present in the form of isolated pools at the GB as shown in Fig. 5, whereas in all castings with cracking, the $\gamma / \gamma^{\prime}$ eutectic is more continuous as shown in Fig. 6. In the optical micrographs [Figs. 5(a) and 5(c)], the bright-appearing microstructural feature between the dendrites is the 


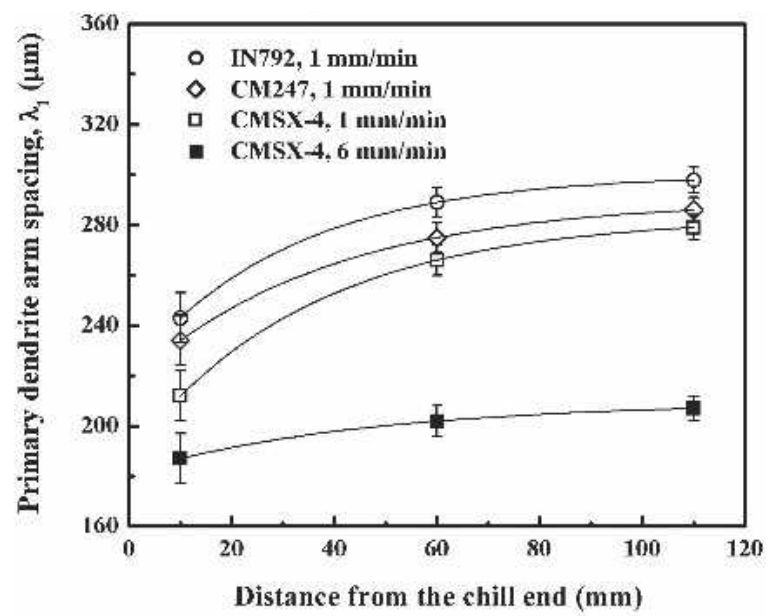

FIG. 4. Primary dendrite arm spacing $\lambda_{1}$ inside the grains measured as a function of the distance from the chill end. $\lambda_{1}$ was determined by counting the number $\left(\mathrm{n}_{1}\right)$ of dendrites within a known area $(A)$ and calculated by the equation: $\lambda_{1}=\left(\mathrm{A} / \mathrm{n}_{1}\right)^{1 / 2}$, i.e., dendrites are assumed to be square shaped. $\lambda_{1}$ increases with increasing distance from the chill end because of change in the thermal gradient and $\lambda_{1}$ of CMSX-4 is smaller at a higher solidification rate.

so-called $\gamma / \gamma^{\prime}$ eutectic, which is an indication of the remaining melt at the end of the solidification process. ${ }^{14}$ The $\gamma / \gamma^{\prime}$ eutectic is discontinuous in the grain interior and at the GB. The interruptions of continuity are marked by open arrows to make them more visible. Figures 5(b) and 5(d) show the detailed microstructure of the GB marked by dark frames in Figs. 5(a) and 5(c), respectively; there is no continuous $\gamma / \gamma^{\prime}$ eutectic at the GB even at the higher magnification. In the castings with small cracks (i.e., small crack ratios), the $\gamma / \gamma^{\prime}$ eutectic is still discontinuous at the GB, but there is more continuity than in the castings without hot cracking.

In samples of CMSX-4 and IN792 that display hot cracking, there is a more or less continuous film of $\gamma / \gamma^{\prime}$ eutectic, even at low levels of GB misorientation. This is shown by Figs. 6(a), 6(c), and 6(e), and the continuous film structure is marked by solid arrows. The detailed microstructure of the GB marked by dark frames in Figs. 6(a), 6(c), and 6(e) is presented in Figs. 6(b), 6(d), and 6(f), respectively. Because the etchant used in the present experiments attacks only $\gamma^{\prime}$ phase of the alloy, it can be deduced that the GB is composed of almost continuous films of $\gamma / \gamma^{\prime}$ eutectic. It is interesting to note that the films of $\gamma / \gamma^{\prime}$ eutectic in CMSX-4 are a little thicker than in IN792 [see Fig. 6(b) and 6(f)].

Figures 5 and 6 allow for another interesting comparison of the $\gamma / \gamma^{\prime}$ eutectic microstructure. It is clear from Figs. 5(a) and 6(a) that the $\gamma / \gamma^{\prime}$ eutectic in CMSX-4 is more finely dispersed, i.e., less continuous, at higher solidification rate. The same result was found in IN792 in our previous work. ${ }^{13}$

The GBs in CM247 are composed of continuous films

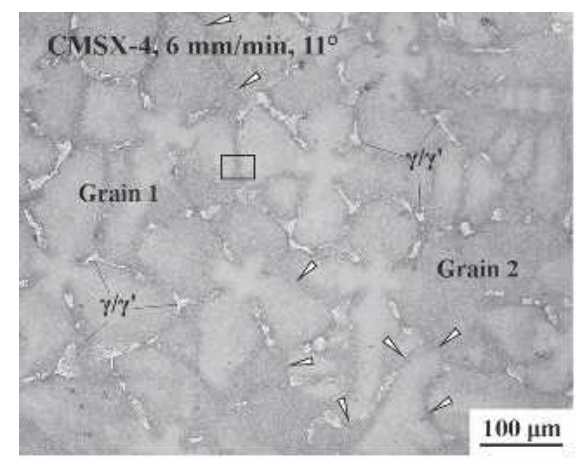

(a)

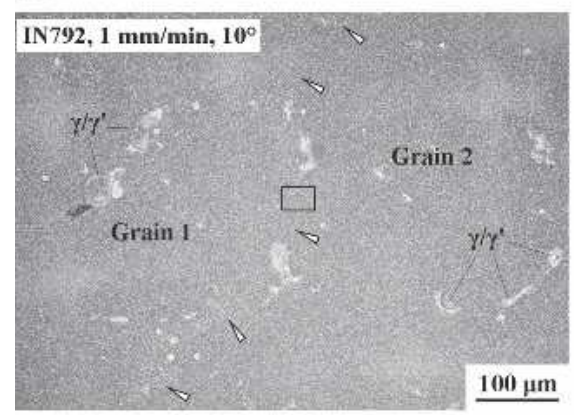

(c)

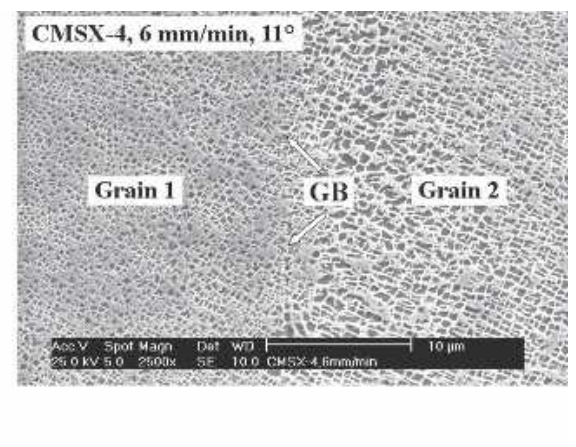

(b)

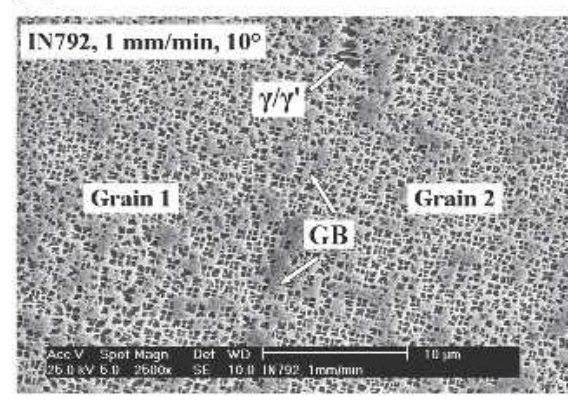

(d)

FIG. 5. GB structure in the castings without hot cracking. (a, c) Optical micrographs showing a general view of the GB and $\gamma / \gamma^{\prime}$ eutectic, and (b, d) SEM images showing the GB structure marked by dark frame in (a) and (c), respectively. At higher magnification, the $\gamma / \gamma^{\prime}$ eutectic is discontinuous at the GB. The discontinuous areas are marked by open arrows in (a) and (c). Although a GB runs right through the center, no continuous film of $\gamma / \gamma^{\prime}$ eutectic is found in (b) and (d). 


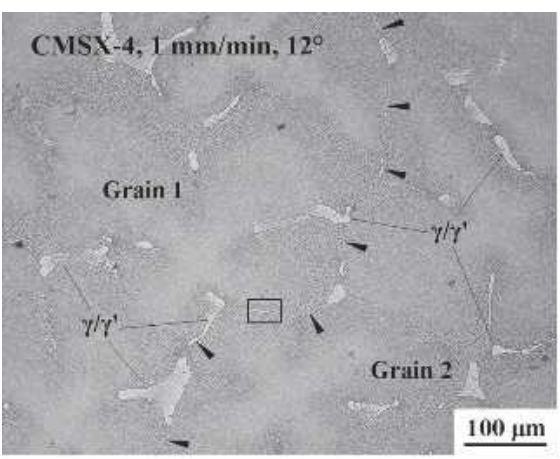

(a)

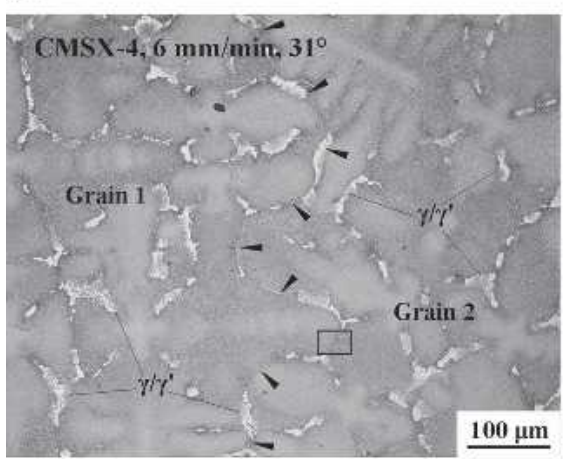

(c)

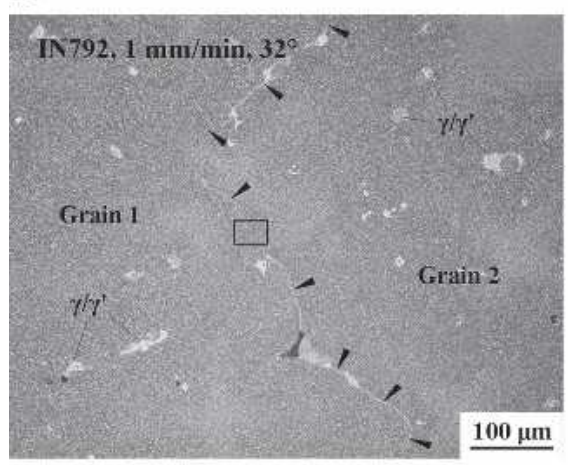

(e)

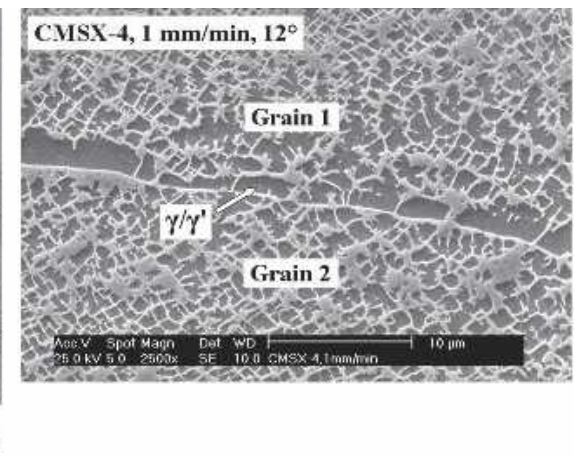

(b)

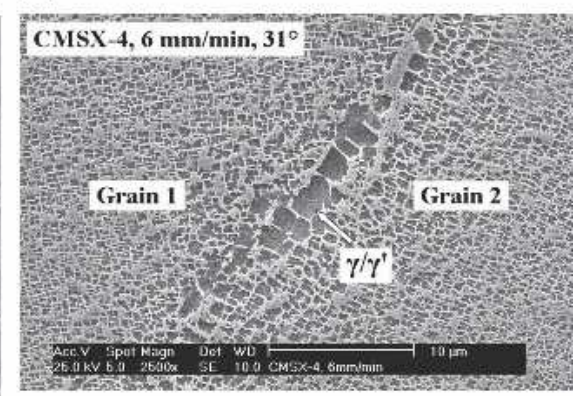

(d)

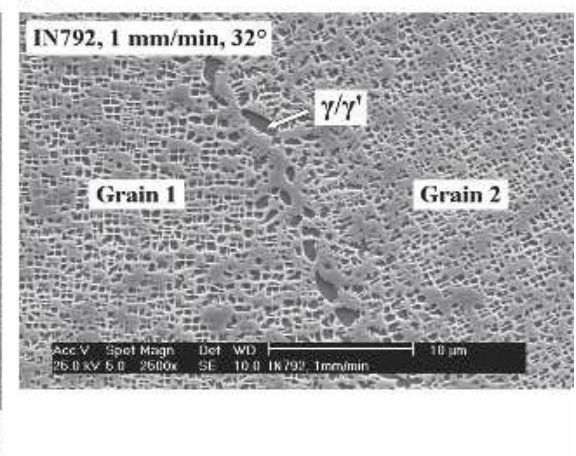

(f)

FIG. 6. GB structure in the castings with hot cracking. (a, c, e) Optical micrographs showing a general view of the GB and $\gamma / \gamma^{\prime}$ eutectic, and (b, d, f) SEM images showing the detailed GB structure marked by dark frame in (a), (c), and (e), respectively. Because the etchant used in the present experiments attacks only $\gamma^{\prime}$ phase in the alloy, it can be deduced that the GBs are composed of continuous films of $\gamma / \gamma^{\prime}$ eutectic. The continuous film structure at the GB is marked by solid arrows in (a), (c), and (e). The amount of $\gamma / \gamma^{\prime}$ eutectic in CMSX-4 is generally higher than in IN792 as shown by (a) and (e).

of $\gamma / \gamma^{\prime}$ eutectic, as well. However, the $\gamma / \gamma^{\prime}$ eutectic films in CM247 are much thicker than in CMSX-4 and IN792 (see Fig. 7). [The magnification of Fig. 7(a), the optical micrograph, is exactly the same as that of the above optical micrographs.] The quantitative evaluation of the thicknesses of $\gamma / \gamma^{\prime}$ eutectic films in different alloys is presented in Fig. 8.

The volume fraction of $\gamma / \gamma^{\prime}$ eutectic is measured as a function of GB misorientation and shown in Fig. 9. The volume fraction of $\gamma / \gamma^{\prime}$ eutectic increases with increasing GB misorientation and the dependence is somewhat stronger at low levels of GB misorientation. The $\gamma / \gamma^{\prime}$ eutectic fraction in the three alloys follows the order: CM247 > CMSX-4 > IN792. The $\gamma / \gamma^{\prime}$ eutectic fraction in CMSX-4 does not change with solidification rate. We found the same for IN792 in our previous work. ${ }^{13}$ The regions divided by dashed lines in Fig. 9 are explained in Sec. IV.

\section{DISCUSSION}

The GB interrupts the regular arrangement of secondary dendrite arms impinging upon each other. Therefore, one may assume that the material cohesion is less strong 


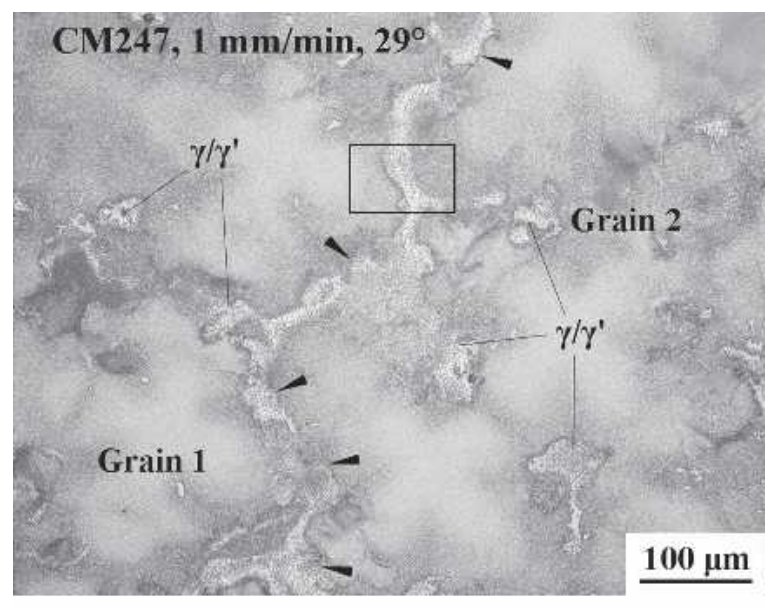

(a)

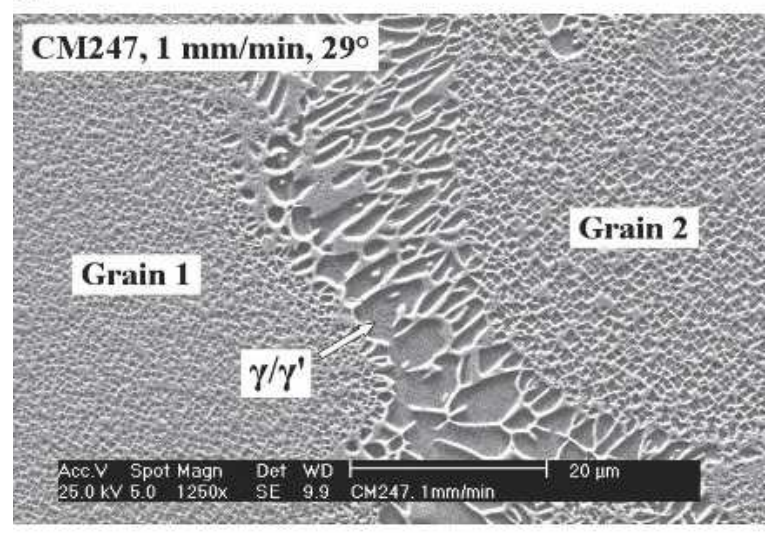

(b)

FIG. 7. GB structure in CM247. (a) Optical micrograph showing a general view of the GB and $\gamma / \gamma^{\prime}$ eutectic and (b) SEM image showing the detailed GB structure marked by dark frame in (a). The continuous film structure at the GB is marked by solid arrows in (a).

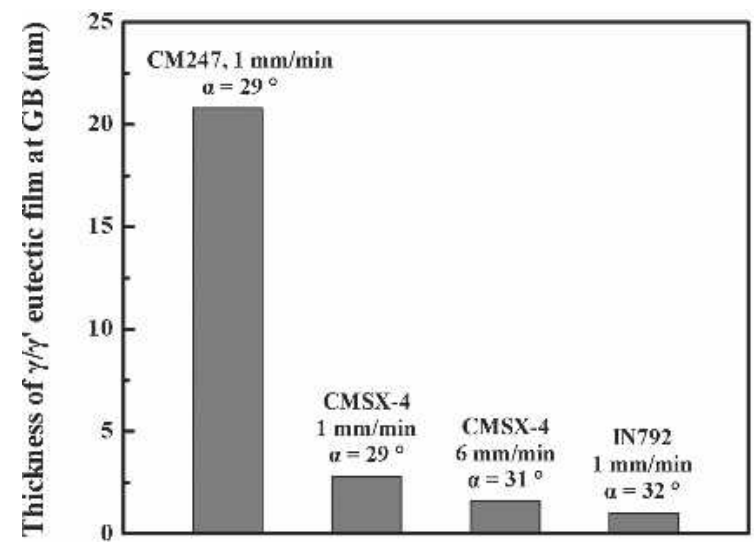

FIG. 8. Comparison of the thicknesses of $\gamma / \gamma^{\prime}$ eutectic films at the GBs in different alloys. CM247 has a much thicker $\gamma / \gamma^{\prime}$ eutectic film.

along the GB because of the reduced bridging and the cohesion strength becomes weaker with increasing GB misorientation. The increase of hot tearing tendency with increasing GB misorientation in CMSX-4 and IN792

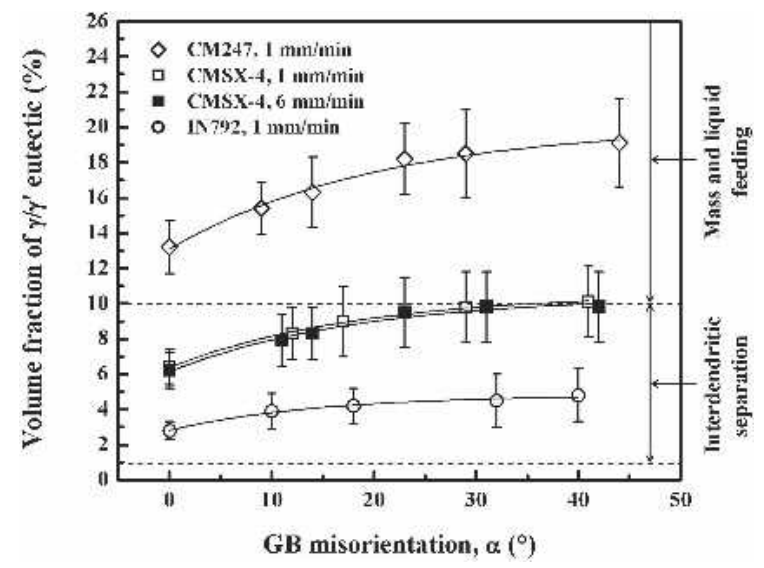

FIG. 9. Volume fraction of $\gamma / \gamma^{\prime}$ eutectic at the GB as a function of GB misorientation. The volume fraction of $\gamma / \gamma^{\prime}$ eutectic increases with increasing GB misorientation, and the increase is rapid at low levels of GB misorientation, but slow at high levels of GB misorientation. Clyne and Davies ${ }^{10}$ defined a critical region of interdendritic separation where the volume fraction of liquid is between $1 \%$ and $10 \%$. The critical region is marked by dashed lines.

corresponds to the decrease of GB cohesion strength with increasing GB misorientation. The different hot tearing susceptibility of different alloys as shown above can be attributed to the different structure of remaining liquid at the GB.

\section{A. Comparison of the castability of CMSX-4, IN792, and CM247}

Clyne and Davies ${ }^{10}$ defined a critical region of interdendritic separation where the volume fraction of liquid is between $1 \%$ and $10 \%$. The critical region is marked by dashed lines in Fig. 9. Because the solidification of Nibased superalloys ends with the $\gamma / \gamma^{\prime}$ eutectic reaction, the volume fraction of $\gamma / \gamma^{\prime}$ eutectic is used to display the volume fraction of the remaining liquid (i.e., eutectic melt) at the last stage of solidification. It is clear from Fig. 9 that the volume fractions of eutectic melt in CMSX-4 and IN792 are within the critical region of interdendritic separation, whereas the volume fractions of eutectic melt in CM247 are within the region that allows mass and liquid feeding. This means that CMSX-4 and IN792 are the alloys with great hot cracking susceptibility according to the general view of hot tearing, whereas CM247 is an alloy with less hot cracking susceptibility because of sufficient feeding.

The volume fraction of eutectic melt is related to the compositions of an alloy and it is an important parameter that dominates dendrite bridging or liquid feeding. Investigations on partitioning behavior of constituent elements showed that $\mathrm{Ta}$ and $\mathrm{Al}$ partition preferentially to the interdendritic regions, whereas $\mathrm{Re}, \mathrm{W}, \mathrm{Co}, \mathrm{Cr}$, and Mo partition to the dendrite core in the high-refractory superalloys. $^{2-4,19-21}$ High $\mathrm{Ta}$ and $\mathrm{Al}$ contents in the 
interdendritic melt enhances the formation of $\gamma / \gamma^{\prime}$ eutectic. ${ }^{19}$ However, the enrichment of Ta in the interdendritic melt can be reduced by carbon additions because of the formation of $\mathrm{TaC} ;{ }^{19-21}$ the $\gamma / \gamma^{\prime}$ eutectic content is therefore reduced. ${ }^{19}$ CMSX-4 has about $70 \%$ higher $\mathrm{Ta}+\mathrm{Al}$ contents than IN792. In particular, CMSX-4 is carbon-free alloy, whereas IN792 is a carbon-containing alloy. Consequently, CMSX-4 forms more $\gamma / \gamma^{\prime}$ eutectic than IN792.

The greater fraction of eutectic melt in CM247 is attributed to the higher $\mathrm{Hf}$ content. Hf is known to promote the formation of $\gamma / \gamma^{\prime}$ eutectic in Ni-based superalloys. ${ }^{22,23}$

The greater fraction of eutectic melt generally corresponds to the thicker liquid film (Fig. 8) and enhances the capability of feeding. Permeability $K$ can be used to describe the degree of feeding of the remaining liquid and $K$ is given by ${ }^{11}$

$$
K=\frac{\lambda_{2}^{2}}{180} \frac{f_{l}^{3}}{\left(1-f_{l}\right)^{2}},
$$

where $\lambda_{2}$ is the secondary dendrite arm spacing and $f_{l}$ is the volume fraction of the remaining liquid (i.e., the volume fraction of eutectic melt in the present work). From the data shown in Fig. 9, the permeability at the GB is a function of GB misorientation shown in Fig. 10. It becomes clear from the figure that the permeability of eutectic melt in CM247 is much greater than in CMSX-4 and IN792. Therefore, sufficient feeding is reasonable at the last stage of solidification in CM247. This explains its very good castability.

Because the eutectic melt in CMSX-4 and IN792 have low permeability at the last stage of solidification (i.e., sufficient feeding cannot be expected at the last stage of

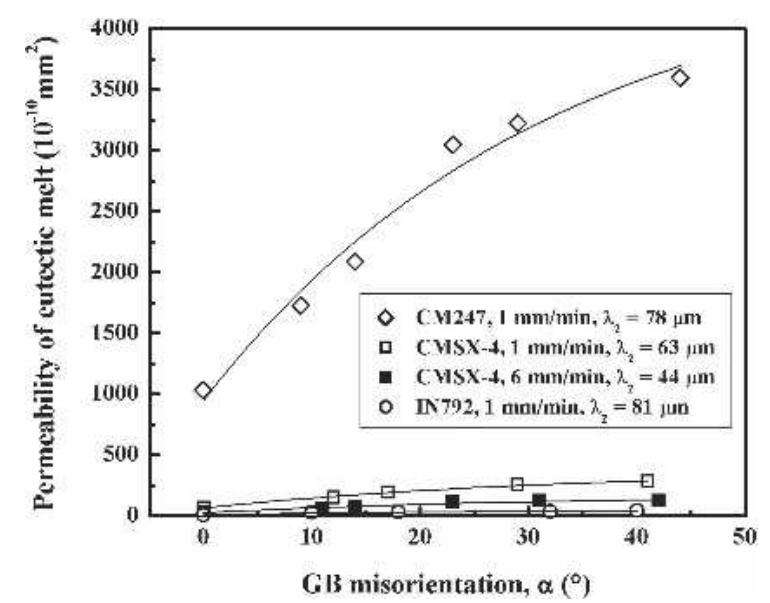

FIG. 10. Permeability of eutectic melt at the GB as a function of GB misorientation. The permeability is low at the last stage of solidification in CMSX-4 and IN792, whereas it is very high in CM247. Sufficient feeding is reasonable at the last stage of solidification in CM247 because of high permeability. solidification in CMSX-4 and IN792), their hot tearing susceptibilities are dominated by GB cohesion strength. A liquid film between the dendrite arms will prevent them from bridging and will make separation easier, before as well as after complete solidification. More $\gamma / \gamma^{\prime}$ eutectic in CMSX-4 tends to develop a more continuous film of liquid and less cohesion strength under conditions where IN792 does not. Therefore, CMSX-4 displays worse castability than IN792.

Figure 11 schematically summarizes the different GB structures and the corresponding castabilities. In Fig. 11(a), good castability, the remaining liquid is present in the form of isolated pools at the GB, and all the castings of CMSX-4 and IN792 without hot cracks correspond to this case. In Fig. 11(b), bad castability, the GB displays a continuously thin film of remaining melt, and all the castings of CMSX-4 and IN792 with hot cracks correspond to the case. In Fig. 11(c), very good castability, the film of the remaining liquid is very thick, and sufficient feeding of liquid is therefore allowed; the castings of CM247 correspond to this case.

\section{B. Influence of solidification rate on castability}

As shown by our results, even the alloy CMSX-4 with its bad castability can be solidified with no cracks if the solidification rate is increased. The reason for the improvement of castability with the solidification rate is the less continuous nature of the $\gamma / \gamma^{\prime}$ eutectic. The reasons for the change in microstructure as a function of solidification rate were discussed in detail in our previous paper. ${ }^{13}$ The important argument is repeated here.

Higher solidification rates produce smaller dendrite arm spacing, which affects $\gamma / \gamma^{\prime}$ eutectic forming. Figure 12 shows a schematic illustration of the impact of dendrite arm spacing on formation of eutectic melt based on the Scheil model, i.e., no diffusion takes place in the solid, and mass transport in the liquid is very rapid. The $\mathrm{c}_{\mathrm{e}}, \mathrm{c}_{\mathrm{sm}}, \mathrm{c}_{0}$, and $\mathrm{k}$ are eutectic composition, composition of maximum solid solution, initial alloy composition, and equilibrium partition ratio, respectively. Solidification begins at the solid concentration of $\mathrm{kc}_{0}$ at the liquidus temperature. The solid composition changes along curve $a b$ during subsequent solidification until its composition reaches $\mathrm{c}_{\mathrm{sm}}$. The remaining liquid has a composition of $\mathrm{c}_{\mathrm{e}}$ at this stage, and eutectic begins to form. To satisfy the mass balance (the average composition of the alloy must be $\mathrm{c}_{0}$ after solidification), the areas of $\mathrm{A}_{1}$ and $\mathrm{A}_{2}$ must be equal. In Fig. 12, the length of line de reflects the size of eutectic; the ratio between the length of line $d e$ and line $c e$ (de/ce) reflects the volume fraction of eutectic. ${ }^{13}$

The dashed curve in Fig. 12 holds for the case of a smaller dendrite arm spacing. As the areas $A_{1}$ and $A_{2}$ decrease with increasing slope of curve $a b^{\prime}$, line $d^{\prime} e^{\prime}$ must be shorter than line de, which means the finer 


\section{Good castability}

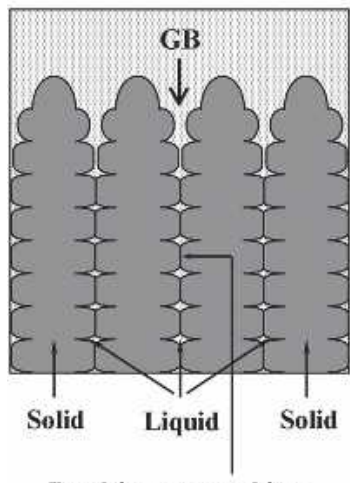

Dendrite arm touching because of isolated pools of liquid

(a)

\section{Bad castability}

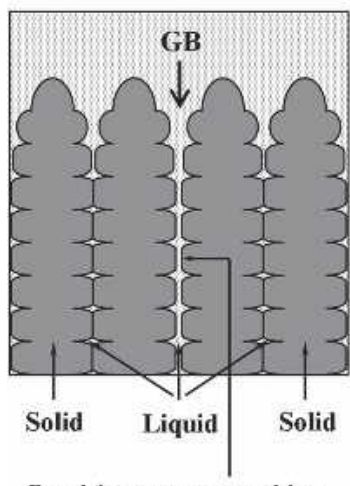

Dendrite arm not touching because of continuously thin liquid film

(b)

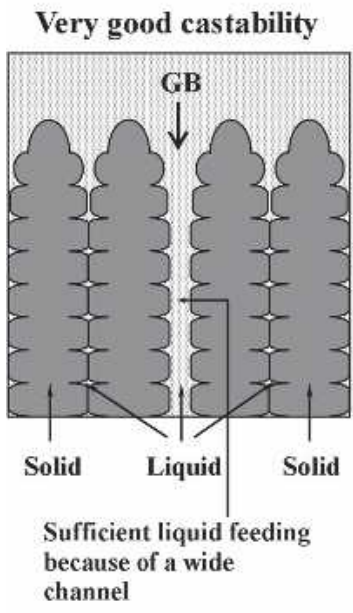

(c)

FIG. 11. Schematic illustration demonstrating the influence of distribution of remaining liquid on castability. (a) The remaining liquid is present in the form of isolated pools at the GB; (b) the remaining liquid forms a continuously thin film at the GB; and (c) the GB displays a continuously thick film of remaining melt. Obviously, GB cohesion is stronger in the first case. Feeding is impeded in second case because of a narrow channel, whereas there is sufficient feeding in the third case because of a wide channel.

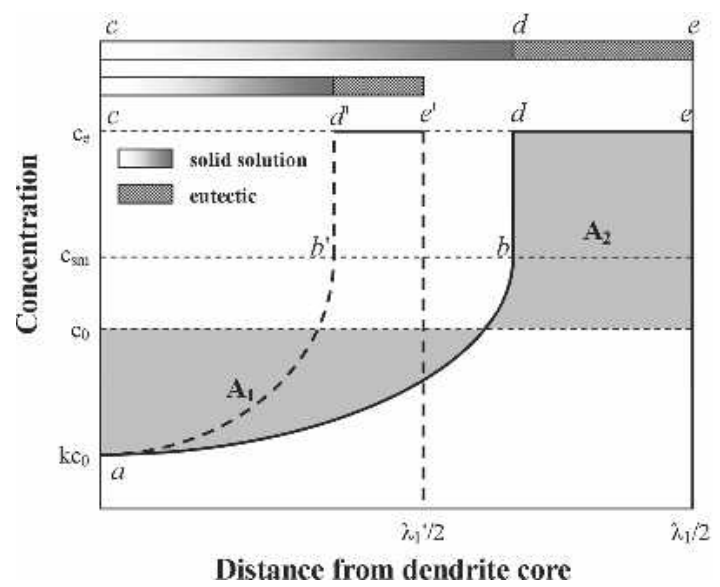

FIG. 12. Schematic illustration of the effect of dendrite arm spacing on formation of eutectic based on a Scheil model from Ref. 13. $\mathrm{c}_{\mathrm{e}}, \mathrm{c}_{\mathrm{sm}}$, $\mathrm{c}_{0}$, and $\mathrm{k}$ are eutectic composition, composition of maximum solid solution, initial alloy composition, and equilibrium partition ratio, respectively. The areas of $\mathrm{A}_{1}$ and $\mathrm{A}_{2}$ are equal. The length of line de reflects the size of eutectic; the ratio of de/ce reflects the volume fraction of eutectic. A shorter dendrite arm spacing (dashed curve) results in finer eutectic. The volume fraction is unaffected.

distribution of the eutectic follows from the reduced dendrite arm spacing. The ratio of de/ce is equal to the ratio of $d^{\prime} e^{\prime} / c e^{\prime}$ in Fig. 12, which means the total volume fraction of eutectic will not change with dendrite arm spacing, i.e., the total volume fraction of eutectic is the same at different withdrawal rates. ${ }^{13}$

Because of the more finely dispersed distribution, the continuous liquid structure at the GB is frequently interrupted because of the bridging of secondary dendrite arms at a high solidification rate, i.e., the GB structure tends to become the first case, as shown in Fig. 11, at a high solidification rate. Consequently, CMSX-4 displays better castability as the solidification rate is increased. This result directly proves that refinement of primary dendrites can reduce the hot tearing susceptibility of DS superalloys.

\section{CONCLUSIONS}

(1) The CMSX-4 SC and BC castings solidified at $1 \mathrm{~mm} / \mathrm{min}$ always showed hot tearing, whereas the IN792 castings solidified at $1 \mathrm{~mm} / \mathrm{min}$ and the CMSX-4 castings solidified at $6 \mathrm{~mm} / \mathrm{min}$ were free of hot cracking at low levels of GB misorientations. These results indicate that the castability of CMSX-4 in terms of hot cracking susceptibility is even worse than that of IN792 and the castability of CMSX-4 can be improved as the solidification rate is increased.

(2) In CMSX-4 and IN792, the eutectic melt is present in the form of isolated pools at the GB in the castings that do not show hot cracking, whereas the eutectic melt forms an almost continuously thin film at the GB in the 
castings that do display hot cracking. These results suggest that the hot tearing susceptibility of CMSX-4 and IN792 is dominated by GB cohesion force.

(3) The film of eutectic melt in CM247 is much thicker than in CMSX-4 and IN792, which allows sufficient feeding of the remaining liquid at the last stage of solidification. The very good castability of CM247 is a consequence of sufficient feeding.

\section{ACKNOWLEDGMENTS}

We thank the German Ministry BMBF for financial support (BMBF Grosser Einkristall 03N2021). Dr. O. Lüsebrink of Doncasters Precision Castings, Bochum, is especially acknowledged for valuable discussions and supplying of material.

\section{REFERENCES}

1. F.L. Versnyder and M.E. Shank: The development of columnar grain and single crystal high temperature materials through directional solidification. Mater. Sci. Eng. 6, 213 (1970).

2. T.M. Pollock and W.H. Murphy: The breakdown of single-crystal solidification in high refractory nickel-base alloys. Metall. Mater. Trans. A 27, 1081 (1996).

3. T.M. Pollock: The growth and elevated temperature stability of high refractory nickel-base single crystals. Mater. Sci. Eng., B 32, 255 (1995).

4. T.M. Pollock, W.H. Murphy, E.H. Goldman, D.L. Uram, and J.S. Tu: Grain defect formation during directional solidification of nickel base single crystals, in Superalloys 1992, edited by S.D. Antolovich, R.W. Stusrud, R.A. MacKay, D.L. Anton, T. Khan, R.D. Kissinger, and D.L. Klarstrom (TMS, Warrendale, PA, 1992), p. 125.

5. N. D'Souza, M.G. Ardakani, M. McLean, and B.A. Shollock: Directional and single crystal solidification of Ni-base superalloys: Part I. The role of curved isotherms on grain selection. Metall. Mater. Trans. A 31, 2877 (2000).

6. M.G. Ardakani, N. D'Souza, B.A. Shollock, and M. McLean: Directional and single crystal solidification of Ni-base superalloys: Part II. Coincidence site lattice character of grain boundaries. Metall. Mater. Trans. A 31, 2887 (2000).

7. M.G. Ardakani, N. D'Souza, A. Wagner, B.A. Shollock, and M. McLean: Competitive grain growth and texture evolution during directional solidification of superalloys, in Superalloys 2000, edited by T.M. Pollock, R.D. Kissinger, R.R. Bowman, K.A. Green, M. McLean, S. Olson, and J.J. Schirra (TMS, Warrendale, PA, 2000), p. 219.

8. G.K. Sigworth: Hot tearing of metals. AFS Trans. 155, 1053 (1996).

9. J. Campbell: Castings (Butterworth-Heinemann Ltd., London, 1991), pp. 219-229.

10. T.W. Clyne and G.J. Davies: The influence of composition on solidification cracking susceptibility in binary alloy systems. Br. Foundrymen 74, 65 (1981).

11. M. Rappaz, J.M. Drezet, and M. Gremaud: A new hot-tearing criterion. Metall. Mater. Trans. A 30, 449 (1999).

12. J. Zhang and R.F. Singer: Effect of grain-boundary characteristics on castability of nickel-base superalloys. Metall. Mater. Trans. A 35, 939 (2004).

13. Y.Z. Zhou, A. Volek, and R.F. Singer: Influence of solidification conditions on the castability of nickel-base superalloy IN792. Metall. Mater. Trans. A 36, 651 (2005).

14. J. Zhang and R.F. Singer: Effect of hafnium on the castability of directionally solidified nickel-base superalloys. Z. Metallkd. 93, 806 (2002).

15. J. Zhang and R.F. Singer: Hot tearing of nickel-based superalloys during directional solidification. Acta Mater. 50, 1869 (2002).

16. V.A. Wills and D.G. McCartney: A comparative study of solidification features in nickel-base superalloys: Microstructural evolution and microsegregation. Mater. Sci. Eng., A 145, 223 (1991).

17. J.E. Hilliard and J.W. Cahn: An evaluation of procedures in quantitative metallography for volume-fraction analysis. Trans. Metall. Soc. AIME 221, 344 (1961).

18. W. Kurz and D.J. Fisher: Dendrite growth at the limit of stability: Tip radius and spacing. Acta Metall. 29, 11 (1981).

19. S. Tin and T.M. Pollock: Stabilization of thermosolutal convective instabilities in Ni-base single-crystal superalloys: Carbide precipitation and Rayleigh numbers. Metall. Mater. Trans. A 34, 1953 (2003).

20. S. Tin, T.M. Pollock, and W. Murphy: Stabilization of thermosolutal convective instabilities in Ni-base single-crystal superalloys: Carbon additions and freckle formation. Metall. Mater. Trans. A 32, 1743 (2001).

21. S. Tin and T.M. Pollock: Phase instabilities and carbon additions in single-crystal nickel-base superalloys. Mater. Sci. Eng., A 348, 111 (2003).

22. D.N. Duhl and C.P. Sullivan: Some effects of hafnium additions on the mechanical properties of a columnar-grained nickel-base superalloys. J. Metall. 23, 38 (1971).

23. J.E. Doherty, B.H. Kear, and A.F. Giamei: On the origin of ductility enhancement in Hf-doped Mar-M200. J. Metall. 23, 59 (1971). 